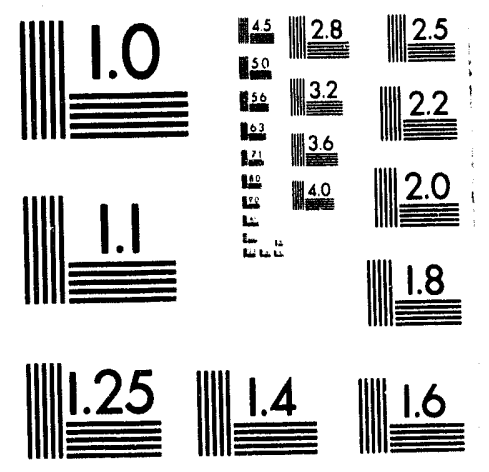



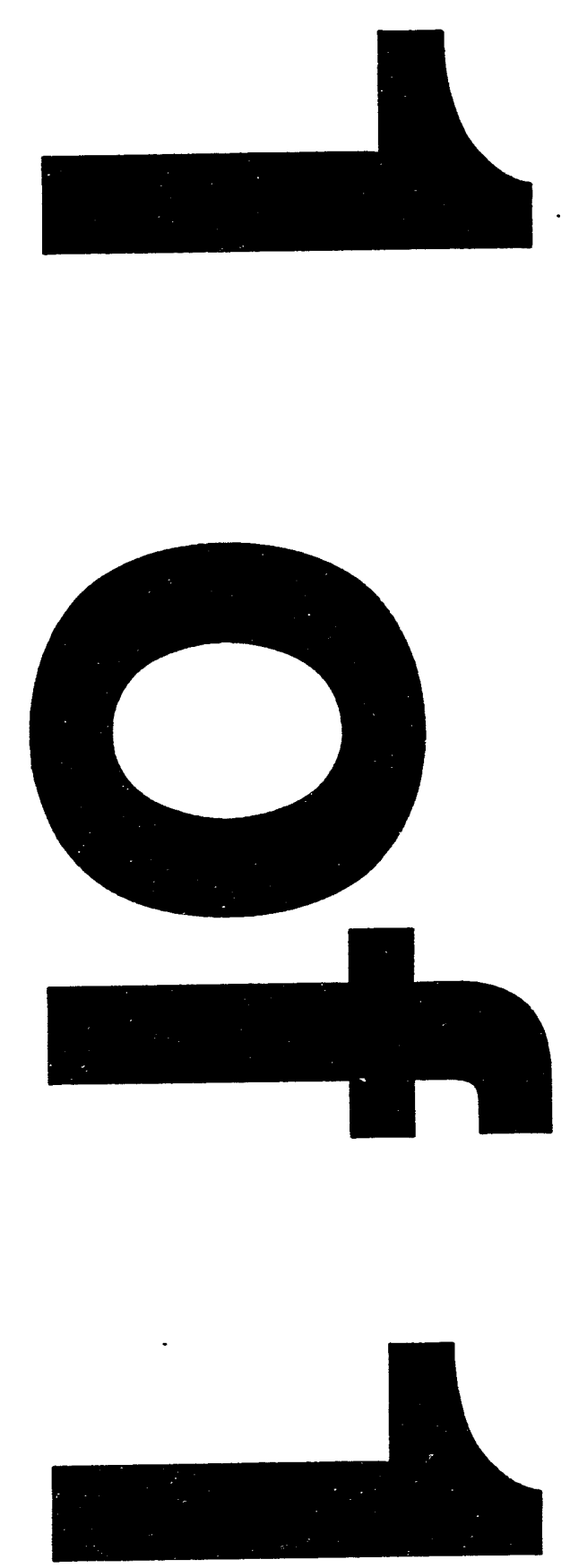

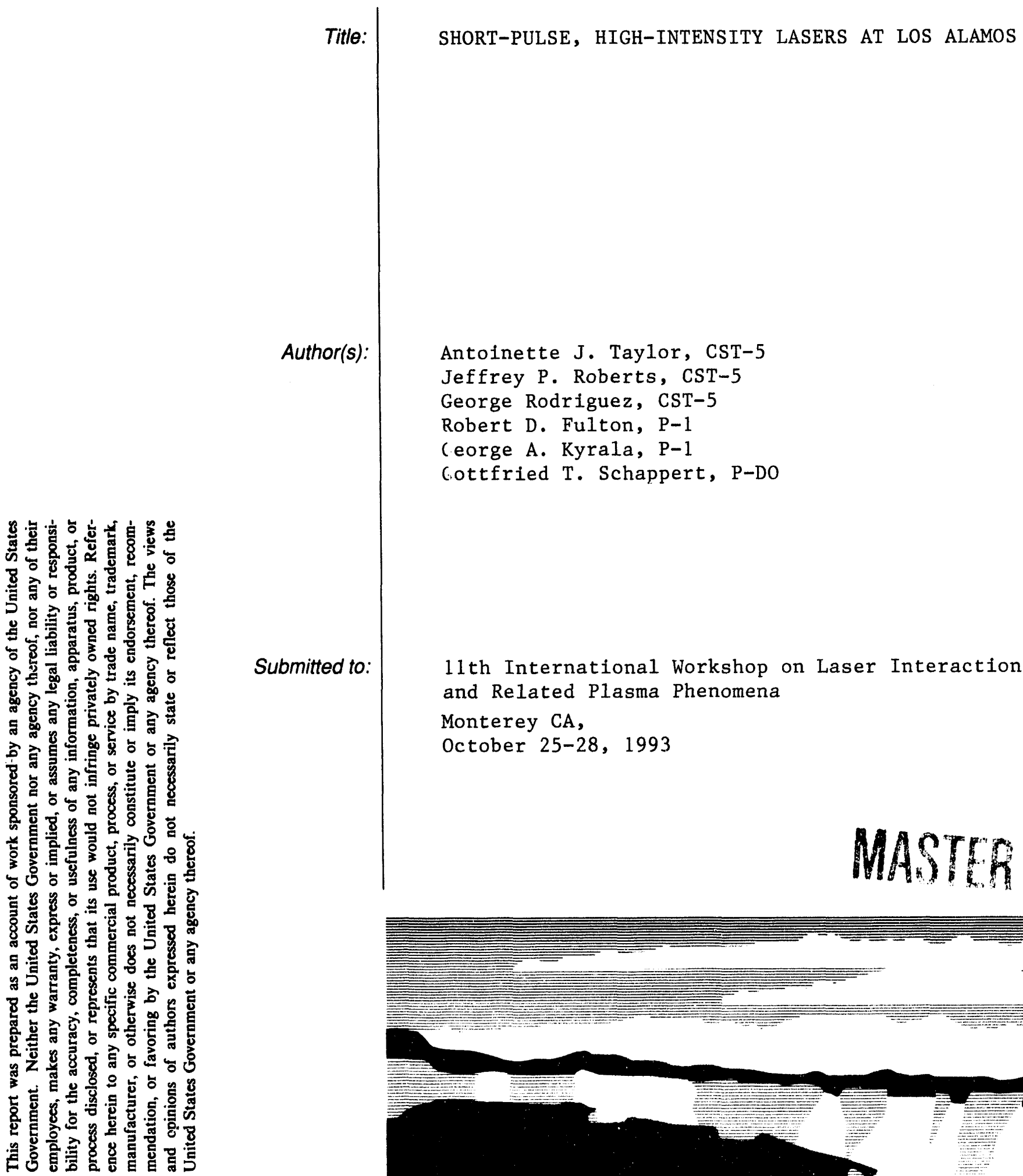

Submitted to:

11 th International Workshop on Laser Interaction and Related Plasma Phenomena

Monterey CA,

October 25-28, 1993

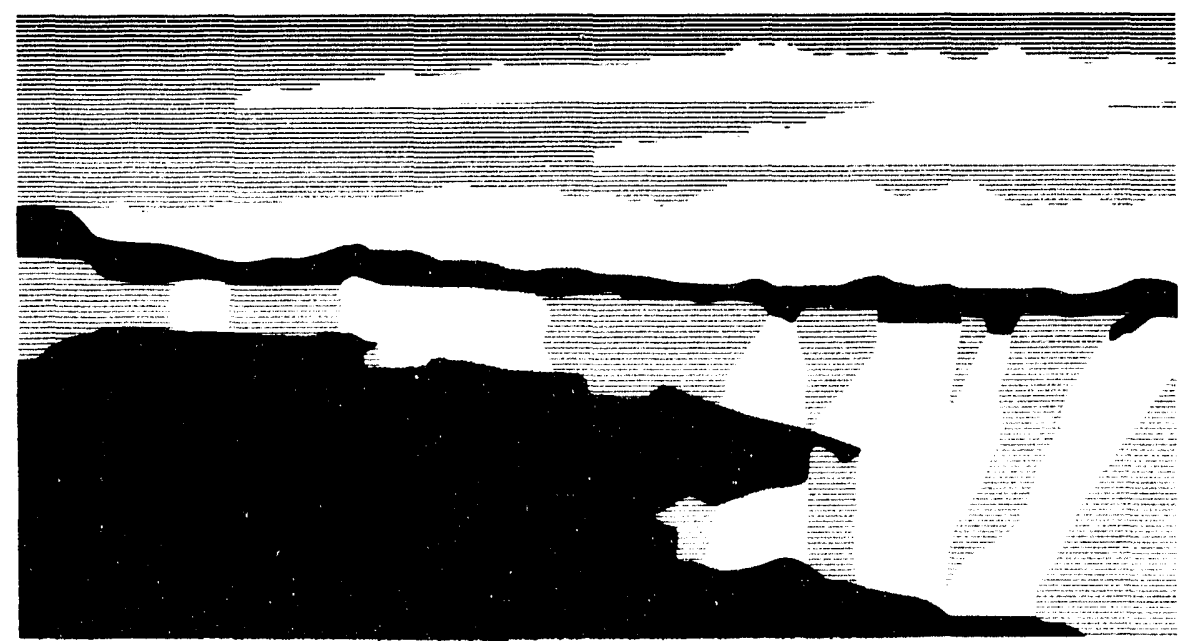

Los Alamos National Laboratory, an affirmative action/equal opportunity employer, is operated by the University of California for the U.S. Department of Energy under contract W-7405-ENG-36. By acceptance of this article, the publisher recognizes that the U.S. Government retains a nonexclusive, royalty-free license to publish or reproduce the published form of this contribution, or to allow others to do so, for U.S. Government purposes. The Los Alamos National Laboratory requests that the publisher identify this article as work performed under the auspices of the U.S. Department of Energy. 


\title{
SHORT-PULSE, HIGH-INTENSITY LASERS AT LOS ALAMOS
}

\author{
A. J. Taylor, J. P. Roberts, G. Rodriguez, R. D. Fulton, G. A. Kyrala \\ and G. T. Schappert \\ Los Alamos National Laboratory \\ MS E543 \\ Los Alamos, NM 87545
}

\begin{abstract}
We describe two laser systems for high intensity laser interaction experiments: The first is a terawatt system based on amplification of femtosecond pulses in $\mathrm{XeCl}$ which yields $250 \mathrm{~mJ}$ in $275 \mathrm{fs}$ and routinely produces intensities on target in excess of $10^{18} \mathrm{~W} / \mathrm{cm}^{2}$. The second system is based on chirped pulse amplification of 100 -fs pulses in Ti:sapphire.
\end{abstract}

\section{INTRODUCTION}

Advances in ultrafast lasers and optical amplifiers have spurred the development of terawatt-class laser systems capable of delivering focal spot intensities approaching $10^{20}$ $\mathrm{W} / \mathrm{cm}^{2}$. At these extremely high intensities, the optical field strength is more than twenty times larger than the Bohr electric field, permitting investigations of the optical properties of matter in a previously unexplored regime. Applications of such laser systems include the study of multiphoton phenomena in atoms, the production and investigation of picosecond $\mathrm{x}$-ray pulses and the testing of optical pumping strategies for $\mathrm{x}$-ray lasers. We describe here two such laser systems for high intensity laser interaction experiments: one based on ultrashort pulse amplification in $\mathrm{XeCl}$ and one based on chirped pulse amplification in Ti:sapphire.

\section{XeCI LASER SYSTEM}


Amplification in $\mathrm{XeCl}$ is the basis of several ultrashort-pulse high-brightness systems worldwide. $(1-4)$ We describe here a terawatt-class laser system based on the amplification of subpicosecond pulses in $\mathrm{XeCl}$ discharge amplifiers.

Generation of 308-nm seed pulses is accomplished by frequency-doubling the output of a synchronously-pumped subpicosecond dye oscillator-amplifier system, ${ }^{(5)}$ operating at 616 $\mathrm{nm}$. These uv seed pulses are amplified in a chain consisting of a small-aperture $\left(1-\mathrm{cm}^{2}\right) \mathrm{XeCl}$ amplifier, a beam-expanding spatial filter and a large aperture $\left(100-\mathrm{cm}^{2}\right)$ final amplifier.

The final amplifier ${ }^{(4)}$ consists of two independently pumped discharge regions, each with clear apertures of $10 \mathrm{~cm}$ by $10 \mathrm{~cm}$. Simultaneous preionization of the gas mixtures in both gain sections is accomplished with a single $130-\mathrm{keV}, 10 \mathrm{mrad} / \mathrm{shot} \mathrm{x}$-ray preionizer. The discharges are driven by low-jitter, thyratron-switched pulse modulators employing two-stage magnetic compression. With $10 \mathrm{kV} / \mathrm{cm} 100 \mathrm{~kW} / \mathrm{cm}^{3}$ discharge pumping and a gas mixture of 2290-Torr Ne, 14-Torr Xe and 1-Torr $\mathrm{HCl}$, the centerline small-signal gain is $0.032 \mathrm{~cm}^{-1}$. Useful gain is available over more than half of the clear aperture. To maintain near-diffractionlimited beam quality at a $1 \mathrm{~Hz}$ repetition rate, a gas flow system is employed that maintains nearly laminar flow transverse to the discharge electrodes. After hot gas is cleared from the discharge volume this flow system establishes less than $\lambda / 20$ wavefront distortion over $80 \%$ of the clear aperture before the next shot is fired.

This laser system generates $250-\mathrm{mJ}, 275-\mathrm{fs}$ pulses at $308 \mathrm{~nm}$ and operates at a sustained repetition rate of $1 \mathrm{~Hz}$. The ultrashort output pulse lies on a 20-ns, 20-mJ amplified spontaneous emission (ASE) pedestal, but only half of the ASE energy is included in the solid angle subtended by the output beam. Using $\mathrm{f} / 3.7$ optics we measure FWHM focal spot dimensions of $3.4 \times 4.1 \mu \mathrm{m}^{2}$ for the full energy final output beam and therefore demonstrate a focal-volume intensity of $6.5 \times 10^{18} \mathrm{~W} / \mathrm{cm}^{2}$.

\section{Ti:SAPPHIRE LASER SYSTEM}

The large gain bandwidth and high saturation fluence of Ti:sapphire ( $\left.\mathrm{Ti}^{\mathrm{A}} \mathrm{Al}_{2} \mathrm{O}_{3}\right)$ permit efficient amplification of ultrashort pulses, allowing Ti:sapphire to become the solid state material of choice for producing ultrahigh peak power pulses. ${ }^{(6-8)}$

Our Ti:sapphire system consists of an oscillator, a chirper, several amplifiers and a pulse compressor. The system is seeded with pulses from a commercial mode-locked 
Ti:sapphire laser operating at $810 \mathrm{~nm}$ and pumped by the 10 Watts, all-lines output of an $\mathrm{Ar}^{+}$ion laser. These 70-fs, 15-nJ pulses have a spectral bandwidth of $9.6 \mathrm{~nm}$. Before amplification, these pulses are temporally stretched using a grating pair pulse-stretcher configured to yield positive group velocity dispersion. (9) Two antiparallel $2000-$ line/mm goldcoated holographic diffraction gratings are separated by $1.7 \mathrm{~m}$, and a unit magnification telescope using 60 -cm-focal-length achromatic lenses is inserted between the gratings. After two passes through the stretcher, the measured pulse duration is $440 \mathrm{ps.} \mathrm{Next,} \mathrm{the} \mathrm{pulses}$ traverse a Faraday isolator. Losses in the beam up to this point (mostly in the stretcher) result in a $5-\mathrm{nJ} /$ pulse energy to seed the amplifier chain.

The Ti:sapphire amplifier chain boosts the energy to $110 \mathrm{~mJ}$ and consists of a regenerative amplifier, a double-pass preamplifier and a final amplifier. All amplifiers are pumped using frequency-doubled Nd:glass/Nd:YAG oscillator/amplifier system. ${ }^{(10)}$ The intensity of these $110-\mathrm{ns}, 0.53-\mu \mathrm{m}$ pump pulses are more than an order of magnitude smaller than those obtained from conventional Q-switched Nd:YAG lasers typically used to pump Ti:sapphire amplifiers, making the system more resistant to optical damage from the pump beam. The strategy used to increase the pump pulse duration is to utilize the low gain of $\mathrm{Nd}$ :glass in the oscillator to yield long-pulse-duration seed pulses at $1.062 \mu \mathrm{m}$, and then use $\mathrm{Nd}$ :YAG, with its superior thermal properties, for amplification up to joule-level energies. The laser system, described in detail in Reference 10, consists of a Q-switched glass oscillator, followed by three Nd:YAG amplifiers operating at a $5 \mathrm{~Hz}$ repetition rate. Frequency-doubling of the output with conversion efficiencies approaching $50 \%$ is achieved in two output arms using a 60-mm-long and a 100-mm-long KD*P crystals.

The first amplifier is a regenerative amplifier using a linear cavity with one flat end mirror and one 3-m focal length end mirror separated by $145 \mathrm{~cm}$ to form a stable TEM 00 mode with a waist size of $1.63 \mathrm{~mm}$ located at the flat end mirror. The $7-\mathrm{mm}$-diameter by $20-\mathrm{mm}$ long Ti:sapphire crystal with $0.15 \%$ Ti doping yields a small signal gain of 4 when pumped with $65 \mathrm{~mJ}$ of $532-\mathrm{nm}$ light focused to a diameter of $2.0 \mathrm{~mm}$. The seed beam is input via a Brewster's reflection off of the Ti:sapphire crystal. A combination of two Pockel's cells, a quarter wave plate and a thin film polarizer is used for pulse selection and switch-out. A 5-ns, 3.6-kV pulse from a pulse generator switches the Pockel's cell. A total of 20 round trips in the cavity is required to boost the pulse energy from $5 \mathrm{~nJ}$ to $7 \mathrm{~mJ}$.

The pulse is then amplified up to the $60-\mathrm{mJ}$ level in a double pass Ti:sapphire preamplifier. The 7 -mm-diameter by $20-\mathrm{mm}$-long, $0.15 \%$ doped, Ti:sapphire rod is pumped 
with $250 \mathrm{~mJ}$ of $532-\mathrm{nm}$ light focused to a $3.0-\mathrm{mm}$-diameter spot. After the first pass the output energy from the Ti:sapphire beam is $28 \mathrm{~mJ}$, and after the second pass, the double pass gain is 8.6 to yield pulse energies of $60 \mathrm{~mJ}$. At the output of the preamplifier, the diameter of the Ti:sapphire beam is $2.9 \mathrm{~mm}$. In order to improve the spatial quality of the output from the Ti:sapphire preamplifier, a vacuum spatial filter is placed between the preamplifier and the final amplifier. The vacuum spatial filter consists of a $125-\mu \mathrm{m}$-diameter diamond pinhole that has a 75\% energy throughput efficiency including reflection losses from telescope lenses and vacuum port windows. The output energy after the vacuum spatial filter is $45 \mathrm{~mJ}$. The telescope for the vacuum spatial filter is also used to expand the Ti:sapphire beam diameter to $4.2 \mathrm{~mm}$ before seeding the final amplifier.

The final amplifier consists of 12 -mm-diameter by 20 -mm-long, $0.15 \%$ doped, Ti:sapphire rod which is pumped with $400 \mathrm{~mJ}$ of green from the second output arm of the 532$\mathrm{nm}$ pump laser. The single pass gain of the final amplifier is 2.6 , yielding an output energy of $110 \mathrm{~mJ}$ for the Ti:sapphire beam. The beam diameters for the pump and the Ti:sapphire beam at this point are $4.4 \mathrm{~mm}$ and $4.2 \mathrm{~mm}$, respectively. Before injecting the amplified Ti:sapphire pulses into the grating compressor, the beam diameter is expanded and collimated to a diameter $9.3 \mathrm{~mm}$ with a dual lens telescope in order to minimize any potential for optical damage to the compressor gratings. After the telescope, the Ti:sapphire pulse energy is $95 \mathrm{~mJ}$.

After, the Ti:sapphire amplifier chain, the pulse width is compressed by a factor of 3200 with a parallel grating pair. The compressor consists of a pair of 2000 lines $/ \mathrm{mm}$ goldcoated holographic gratings separated by $74 \mathrm{~cm}$. Although compressed pulse widths of $110 \mathrm{fs}$ are achievable, day-to-day operation of the system yields a typical compressed pulse width of $125 \mathrm{fs}$. The throughput energy efficiency of the grating pair compressor is $45 \%$, and the output pulse energy of the compressed beam is $43 \mathrm{~mJ}$. The spectral width of $8.8 \mathrm{~nm}$ for the compressed pulse yields a time-bandwidth product of $\Delta v \Delta t=0.443$. Upon measuring the output energy stability at a repetition frequency of $5 \mathrm{~Hz}$, the pulse-to-pulse amplitude stability is within $12 \%$.

\section{ACKNOWLEDGEMENTS}

We acknowledge the valuable technical assistance of Keith Hosack in the development of the laser systems described here. 


\section{REFERENCES}

1. J. H. Glownia, G. Arivalingam, P. P. Sorokin and J. E. Rothenberg, "Amplification of 350-fsec pulses in XeCl excimer gain modules," Opt. Lett. 11, 79 (1986).

2. S. Watanabe, A. Endoh, M. Wantanabe and N. Surakura, " Terawatt XeCl discharge laser system," Opt. Lett. 13, 580 (1988).

3. S. A. Akhamanov, V. M. Gordienko, M. S. Dzhidshoev, S. V. Krayushkin, I. A. Kudinov, V. T. Platonenko, and V. P. Popov, "Generation and amplification of subpicosecond ultraviolet radiation pulses in excimer lasers," Sov. J. Quantum Electron. 16, 1291 (1987).

4. A. J. Taylor, C. R. Tallman, J. P. Roberts, C. S. Lester, T. R. Gosnell, P. H. Y. Lee and G. A. Kyrala, "High-intensity, subpicosecond XeCl laser system," Opt. Lett. 15, 39 (1990).

5. A. J. Taylor, J. P. Roberts, T. R. Gosnell and C. S. Lester, "Synchronously pumped subpicosecond dye oscillator-amplifier system," Opt. Lett. 14, 444 (1989).

6. J. Squier, F. Salin, G. Mourou and D. Harter, "100-fs pulse generation and amplification in Ti:Al2 $\mathrm{O}_{3}, "$ Opt. Lett. 16, 324 (1991).

7. J. D. Kmetec, J. J. Macklin and J. F. Young, " 0.5-TW, 125-fs Ti:sapphire laser," Opt. Lett. 16, 1001 (1991).

8. C. Le Blanc, G. Grillon, J. P. Chambaret, A. Migus and A. Antonetti, "Compact and efficient multipass Ti:sapphire system for femtosecond chirped-pulse amplification at the terawatt level," Opt. Lett. 18, 140 (1993).

9. O. E. Martinez, " 3000 times grating compressor with positive group velocity dispersion: applications to fiber compensation in the 1.3 to $1.6 \mu \mathrm{m}$ region," IEEE J. Quantum Electron. QE-23, 59 (1987).

10. J. P. Roberts, K. W. Hosack, A. J. Taylor, J. Weston and R. N. Ettlebrick, "Efficient frequency-doubled long-pulse generation with a Nd:glass/Nd:YAG oscillator-amplifier system," Opt. Lett. 18, 429 (1993). 

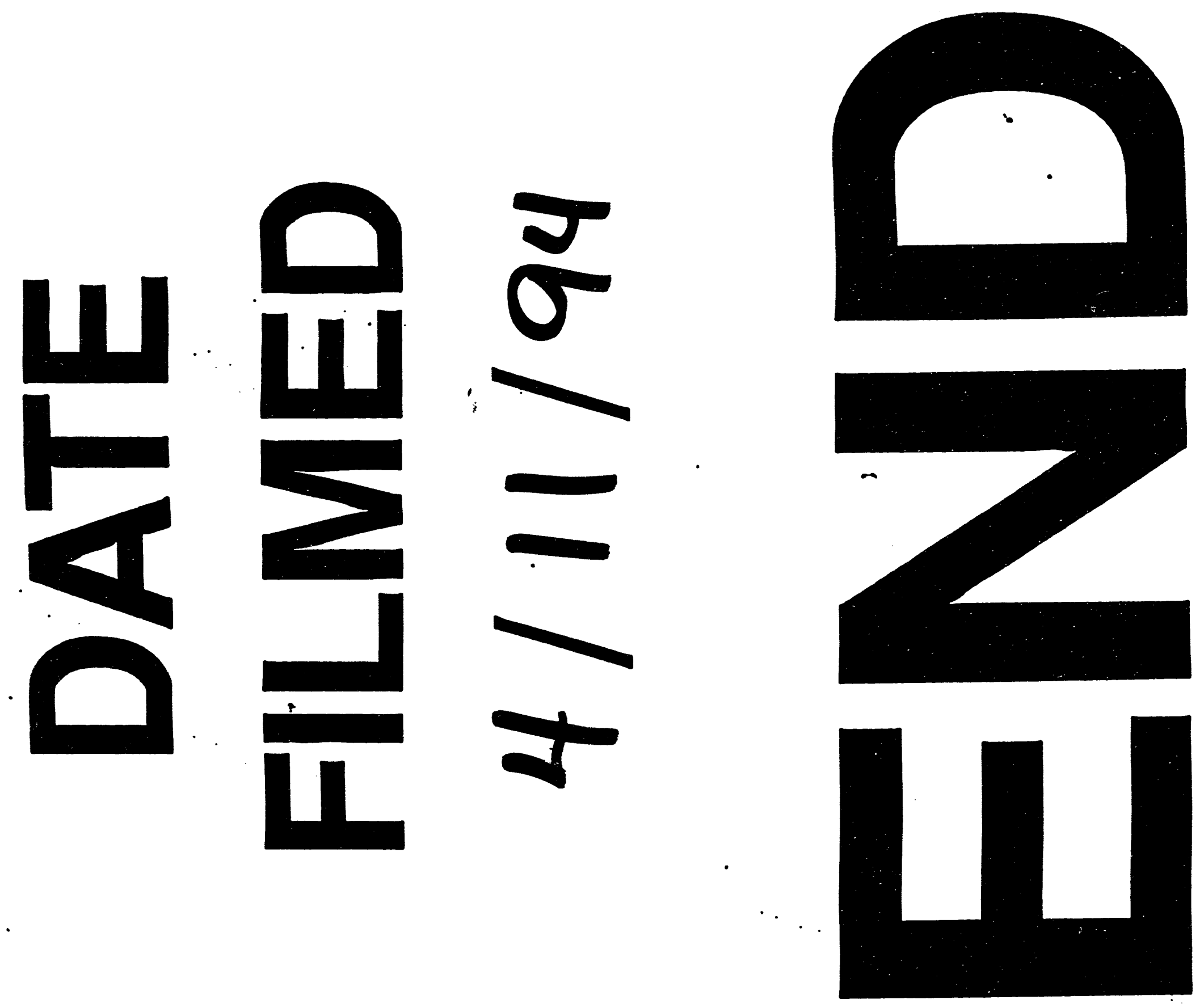
